Hans Moons, Hemantbhai H. Patel, Sergiu M. Gorun and Sabine Van Doorslaer*

\title{
Electron Paramagnetic Resonance and DFT Analysis of the Effects of Bulky Perfluoroalkyl Substituents on a Vanadyl Perfluoro Phthalocyanine
}

DOI 10.1515/zpch-2016-0827

Received June 13, 2016; accepted October 10, 2016

Abstract: $\mathrm{F}_{64} \mathrm{PcVO}$, the vanadyl complex of the perfluoro-isopropyl-substituted perfluorophthalocyanine ligand $\mathrm{F}_{64} \mathrm{PcH}_{2}$, exhibits interesting electronic properties compared to related vanadyl phthalocyanine complexes. X-and W-band continuous-wave electron paramagnetic resonance (CW EPR) of undiluted $\mathrm{F}_{64} \mathrm{PcVO}$ powders illustrate the absence of $\pi-\pi$ stacking in these materials due to the bulky substituents. Furthermore, pulsed EPR and electron nuclear double resonance (ENDOR) experiments in combination with density functional theory (DFT) computations were used to determine the principal $g$ values and the ${ }^{51} \mathrm{~V},{ }^{14} \mathrm{~N},{ }^{1} \mathrm{H}$ and ${ }^{19} \mathrm{~F}$ hyperfine couplings of a frozen ethanol solution of $\mathrm{F}_{64} \mathrm{PcVO}$. The axial ligation of ethanol to vanadium was proven. Axial accessibility of the vanadyl center combined with the high solubility of the complex in different solvents make $\mathrm{F}_{64} \mathrm{PcVO}$ an interesting molecule for catalytic applications.

Keywords: DFT; EPR; fluorinated phthalocyanine; Phthalocyanine; vanadyl.

Dedicated to: Kev Salikhov on the occasion of his 80th birthday.

\section{Introduction}

Phthalocyanines (Pc) and metal phthalocyanines (PcM) have a large range of applications. For example, they are used as dyes and pigments, as catalysts and

\footnotetext{
*Corresponding author: Sabine Van Doorslaer, BIMEF Laboratory, Department of Physics, University of Antwerp, Universiteitsplein 1, B-2610 Antwerp, Belgium, e-mail: sabine.vandoorslaer@uantwerpen.be

Hans Moons: BIMEF Laboratory, Department of Physics, University of Antwerp, Universiteitsplein 1, B-2610 Antwerp, Belgium

Hemantbhai H. Patel and Sergiu M. Gorun: Department of Chemistry and Biochemistry and Center for Functional Materials, Seton Hall University, South Orange, NJ 07079, USA
} 
as non-linear optical materials, and are promising materials for photodynamic therapy, for gas sensing and for new solar-cell applications [1, 2]. While planar phthalocyanines readily form $\pi-\pi$ stacks, which hamper solubility and singlesite accessibility necessary for catalysis, introduction of bulky ring substituents can reduce this stacking (due to steric hindrance). The ring substituents can also strongly influence the electronic properties of the phthalocyanines. Electronwithdrawing substitutents lead to easily reducible Pc complexes, as is illustrated by the fact that $\mathrm{F}_{16} \mathrm{PcM}$ are $n$-type semiconductors as thin films.

Recently, some of us have introduced a new class of metallo phthalocyanines with extreme electron deficiency by placing eight $i-C_{3} F_{7}$ peripheral substituents in the perfluorophthalocyanine ring leading to so-called perfluoroalkylated perfluorophthalocyanines, i.e the $29 H, 31 H, 1,4,8,11,15,18,22,25$-octafluoro2,3,9,10,16,17,23,24-octakis-perfluoro-isopropyl, $\mathrm{F}_{64} \mathrm{PcH}_{2}[3]$ and its different metal complexes (Figure 1a) [4-7]. These materials exhibit enhanced solubility and interesting electronic and catalytic properties.

In the current report, we focus on the characterization of the vanadyl complex $\mathrm{F}_{64} \mathrm{PcVO}$. It was shown earlier that, despite the large molecular weight of this phthalocyanine, its vapor deposition is facile and leads to intensely colored thin films [6]. Furthermore, in vitro electrical conduction and optical transmission measurements suggested that the intermolecular electronic coupling is neglible [6]. Single-crystal X-ray structures of $\mathrm{F}_{64} \mathrm{PcVO}$ revealed hexacoordination of the vanadium center, with an axially ligated water molecule trans to the $\mathrm{V}=\mathrm{O}$ bond [8], Figure 1d. Furthermore, these experiments revealed that the fluoroalkyl ligand substitutents strongly hinder $\pi-\pi$ stacking with single-site isolation, and favor long-range packing [8]. In the present work, we investigate both $\mathrm{F}_{64} \mathrm{PcVO}$ powders and frozen ethanol solutions of $\mathrm{F}_{64} \mathrm{PcVO}$ using multi-frequency continuous wave (CW) and pulsed electron paramagnetic resonance (EPR) spectroscopy in combination with density functional theory (DFT) computations. We analyse how the solid-state arrangement influences the spin-spin interactions usually observed in phthalocyanine powders and study whether the axial ligation to $\mathrm{F}_{64} \mathrm{PcVO}$ persists in solution.

\section{Experimental}

\subsection{Materials}

1,4,8,11,15,18,22,25-octafluoro-2,3,9,10,16,17,23,24-octakis-perfluoro-isopropyl vanadyl(II) phthalocyanine, $\mathrm{F}_{64} \mathrm{PcVO}$, was prepared as reported previously [6]. 
(a)

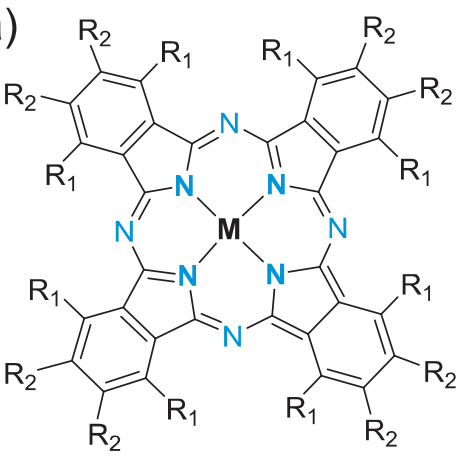

(c)

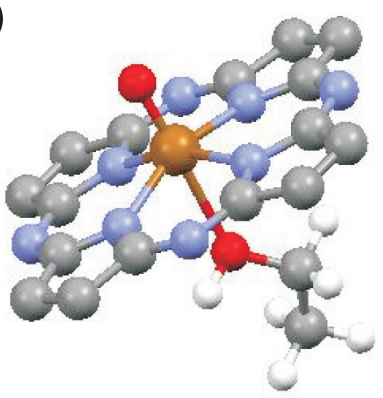

(b)

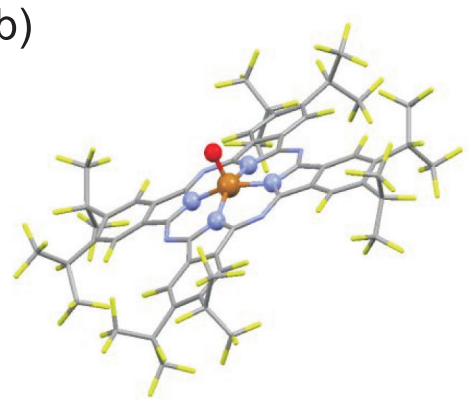

(d)

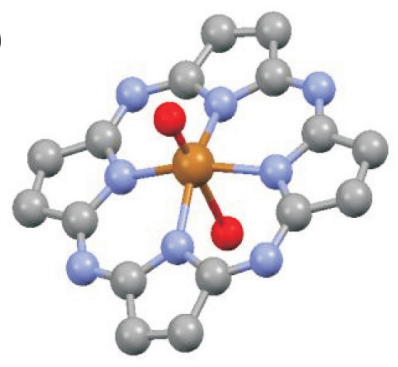

Fig. 1: (a) Structural formula for $F_{16} P c V O\left(M=[V(I V) O]^{2+}, R_{1}=R_{2}=F\right)$ and $F_{64} P c V O\left(M=[V(I V) O]^{2+}\right.$, $\left.R_{1}=F, R_{2}=C F\left(C_{3}\right)_{2}\right)$. (b) Stick representation of the DFT-optimized structure of $F_{64} P c V O(E t O H)$ (model D). Only a truncated 5 -coordinated vanadium $\mathrm{N}_{4} \mathrm{O}$ core coordination sphere is shown in balls representation. The coordinated solvent is omitted for the sake of clarity. (c) Ball-and-stick representation of the $\mathrm{N}_{4} \mathrm{~V}=\mathrm{O}$ (ethanol) coordination sphere and a truncated macrocycle. (d) Ball-and-stick representation of the Vanadium $\mathrm{N}_{4} \mathrm{O}_{2}$ coordination sphere of 6-coordinated $\mathrm{F}_{64} \mathrm{PcVO}\left(\mathrm{H}_{2} \mathrm{O}\right)$ (X-ray structure). See also Table 2. $\mathrm{H}$ atoms have been omitted from the $\mathrm{X}$-ray structure for the sake of clarity. Color code: $\mathrm{V}$ orange, $\mathrm{N}$ blue, $\mathrm{O}$ red, $\mathrm{C}$ gray, $\mathrm{H}$ white.

Vanadyl 2,9,16,23-tetraphenoxy-29H,31H-phthalocyanine ( $\left.\mathrm{Pc}^{t p} \mathrm{VO}\right)$ was obtained from Sigma Aldrich ( $98 \%$ dye content).

Solvent-free $\mathrm{F}_{64} \mathrm{PcVO}$ is dissolved in ethanol or deuterated ethanol- $\mathrm{d}_{6}$ to a concentration of $\sim 1 \mathrm{mM}$. All reagents are obtained from commercial sources and used without purification. Isotopically enriched solvents (99\%) are obtained from Cambridge Isotopes Laboratories.

All reagents were used without further purification. 


\subsection{Electron paramagnetic resonance and UV-Vis spectroscopy}

X-band continuous-wave (CW) EPR spectra were obtained using a Bruker ESP300E spectrometer (microwave (mw) frequency of $9.43 \mathrm{GHz}$ ) equipped with a liquid-helium cryostat (Oxford Inc.), allowing for operation from room temperature to $2.5 \mathrm{~K}$. The presented CW-EPR spectra are recorded at room temperature. The spectra are recorded with a magnetic field modulation of $0.5 \mathrm{mT}$ and $100 \mathrm{kHz}$ modulation frequency. The microwave power was $2 \mathrm{~mW}$.

All X-band pulsed EPR experiments were recorded on a Bruker Elexsys E580 spectrometer (mw frequency of $9.76 \mathrm{GHz}$ ) equipped with a liquid-helium cryostat (Oxford Inc.). All experiments were performed with a repetition rate of $1 \mathrm{kHz}$.

The X-band electron spin echo (ESE)-detected EPR experiments were performed at $10 \mathrm{~K}$ with an mw pulse sequence $\pi / 2-\tau-\pi-\tau$-echo with $t_{\pi / 2}=16 \mathrm{~ns}, t_{\pi}=32$ ns and $\tau=176 \mathrm{~ns}$.

Mims ENDOR (electron nuclear double resonance) spectra [9] were recorded with an mw pulse sequence $\pi / 2-\tau$ - $\pi / 2-T-\pi / 2-\tau$-echo with $t_{\pi / 2}=16$ ns and $T=17 \mu \mathrm{s}$. The $\tau$-value is changed from $96 \mathrm{~ns}$ to $736 \mathrm{~ns}$ in steps of $16 \mathrm{~ns}$ and the corresponding spectra were summed to avoid blind spots in the spectrum. A radio-frequency (rf) pulse of $17 \mu \mathrm{s}$ with variable frequency is applied during the time interval $T$ with an rf increment of $23 \mathrm{kHz}$. The rf pulse length is optimized for ${ }^{1} \mathrm{H}$ nuclei.

The HYSCORE (hyperfine sublevel correlation) spectra [10] were measured at $10 \mathrm{~K}$ using a pulse sequence $\pi / 2-\tau-\pi / 2-t_{1}-\pi-t_{2}-\pi / 2-\tau$-echo, with pulse lengths $t_{\pi / 2}=16$ ns and $t_{\pi}=32 \mathrm{~ns}$. Two $\tau$ values (96 ns and $176 \mathrm{ns)}$ ) were used. The time intervals $t_{1}$ and $t_{2}$ were varied from $96 \mathrm{~ns}$ to $4896 \mathrm{~ns}$ in steps of $16 \mathrm{~ns}$. A four-step phase cycle was used to eliminate unwanted echoes.

All W-band CW and pulsed EPR experiments were performed using a Bruker Elexsys E680 spectrometer (mw frequency $\sim 94 \mathrm{GHz}$ ), equipped with a continuous flow cryostat and a superconductive magnet from Oxford, Inc. The CW EPR spectrum was recorded at room temperature with a modulation amplitude of 0.5 $\mathrm{mT}$ and a mw power of $0.044 \mathrm{~mW}$. The W-band ESE-detected EPR experiments were performed at $10 \mathrm{~K}$ with an mw pulse sequence $\pi / 2-\tau$ - $\pi$ - $\tau$-echo with $t_{\pi / 2}=148$ $\mathrm{ns}, t_{\pi}=296 \mathrm{~ns}$ and $\tau=900 \mathrm{~ns}$.

Simulations of the EPR and HYSCORE spectra were done with the EasySpin program, a MATLAB toolbox developed for EPR simulations [11]. The HYSCORE data were processed with MATLAB 7.7.0 (The MathWorks, Inc., Natick, MA, USA). The time traces were baseline-corrected with a third-order polynomial, apodized with a Hamming window and zero-filled. After a two-dimensional Fourier transformation, the absolute spectra were computed. Spectra recorded with different $\tau$ values were added in the frequency domain after normalizing to the noise level to eliminate blind-spot effects. 
UV-visible absorption spectra were recorded on a Cary 500 Scan UV-Vis-NIR spectrophotometer using a $1 \mathrm{~mm}$ quartz cell and dry ethanol as a solvent.

\subsection{DFT computations}

Spin-unrestricted DFT computations were performed with the ORCA package [12-15] on different $\mathrm{F}_{64} \mathrm{PcVO}$ and $\mathrm{F}_{64} \mathrm{PcVO}$ (ethanol) models. Model A is $\mathrm{F}_{64} \mathrm{PcVO}$ in vacuum, model $\mathrm{B}$ is $\mathrm{F}_{64} \mathrm{PcVO}$ in ethanol, model $\mathrm{C}$ is $\mathrm{F}_{64} \mathrm{PcVO}$ (ethanol) in vacuum and model $\mathrm{D}$ is $\mathrm{F}_{64} \mathrm{PcVO}$ (ethanol) in ethanol (Figure $1 \mathrm{~b}$ and $\mathrm{c}$ ). The vanadium coordination numbers (CN) for these models are 5, 5, 6 and 6, respectively. To simulate the solvent, a dielectric surrounding with the dielectric constant of ethanol according to the COSMO model is used [16]. For the geometry optimizations, the Becke-Perdew density functional (BP86) [17-19] is used together with the resolution of the identity method [20-22]. The split-valence plus polarization (SVP) basis set [23] is used for all atoms except for the vanadium atom a more polarized triple zeta valence (TZVPP) basis is used (Ahlrichs, unpublished). The energy is converged to $1 \times 10^{-8}$ Hartree (Eh) and the convergence tolerances in the geometry optimization are $3 \times 10^{-4} \mathrm{Eh} /$ Bohr for the gradient and $5 \times 10^{-6}$ Eh for the total energy. The coordinates of the optimized geometries are given in the supplementary material. Single-point calculations with the B3LYP functional [24] were carried out at the optimized geometries to predict the EPR spectral parameters. In these calculations the same basis sets were used as in the geometry optimization except for the nitrogen atoms, the pyrrole carbon atoms, the oxygen atoms and the closest hydrogen atom, the Barone EPR-II basis set was used [25] and for vanadium the triply polarized "Core properties" [CP(PPP)] was used ([26] and R. Ahlrich and co-workers, unpublished).

\section{Results and discussion}

When comparing the room-temperature X-band CW EPR spectra of undiluted powders of $\mathrm{F}_{64} \mathrm{PcVO}$ powder and vanadyl 2,9,16,23-tetraphenoxy-29H,31H-phthalocyanine ( $\mathrm{Pc}^{t p} \mathrm{VO}$ ) (Figure $2 \mathrm{a}$ and $\mathrm{b}$ ), a clear difference in the spectral features can be observed. While the latter phthalocyanine gives rise to a broad EPR line, the EPR spectrum of the $\mathrm{F}_{64} \mathrm{PcVO}$ powder shows nicely resolved lines. Although the tetraphenoxy substituents of $\mathrm{Pc}^{t p} \mathrm{VO}$ are expected to partially prevent $\pi$ - $\pi$ stacking of the phthalocyanines, the broad feature indicates that there is still a high degree of dipolar broadening due to the close proximity of the metal centers. 

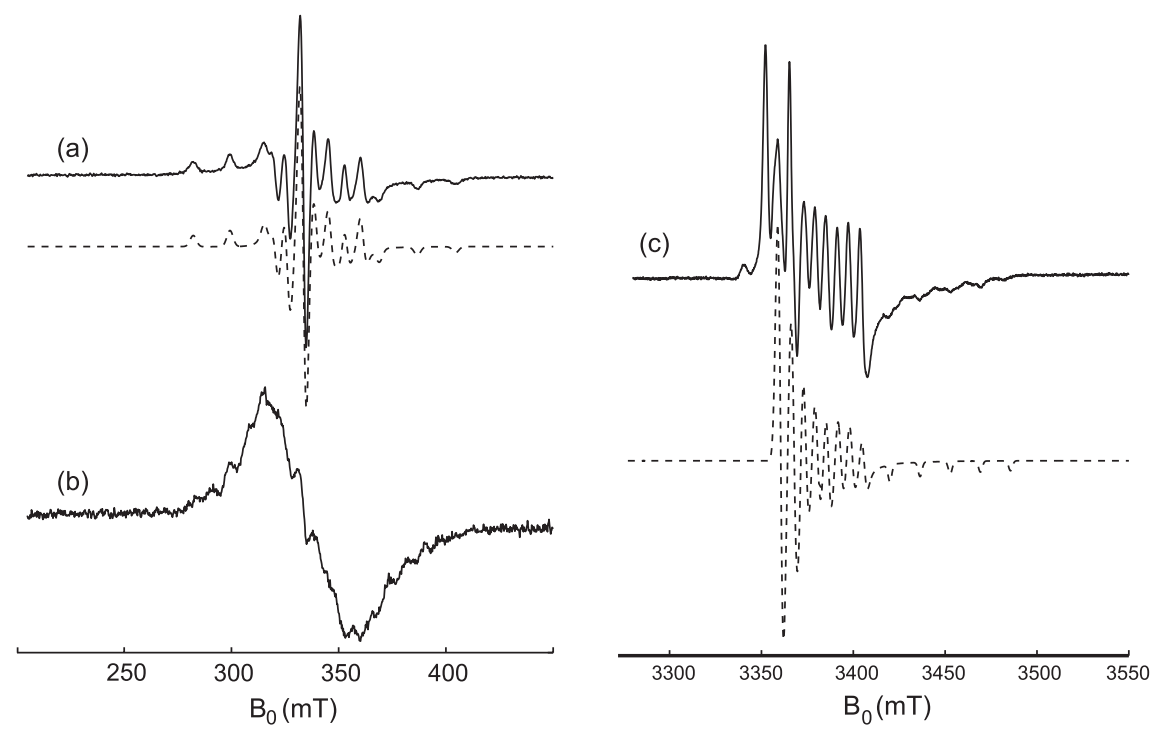

Fig. 2: Experimental (solid line) and simulated (dashed line) X-band ( $a, b)$ and W-band (c) CW-EPR spectra of $\mathrm{F}_{64} \mathrm{PcVO}$ powder $(\mathrm{a}, \mathrm{c})$ and $\mathrm{Pc}^{t p} \mathrm{VO}(\mathrm{b})$ recorded at room temperature. The simulation parameters are given in Table 1.

This strong dipolar broadening is not observed for $\mathrm{F}_{64} \mathrm{PcVO}$, indicating a larger distance between the different vanadyl centers. This corroborates the earlier $\mathrm{XRD}$ finding for $\mathrm{F}_{64} \mathrm{PcVO}$, that show long-range order without $\pi-\pi$ stacking [8]. A similar observation was noted for $\mathrm{F}_{64} \mathrm{PcCu}[7]$.

A higher resolution of the EPR spectrum of the $\mathrm{F}_{64} \mathrm{PcVO}$ powder can be achieved at W-band mw frequencies (Figure 2c). The spectrum is typical of a magnetically isolated vanadyl complex ( $\mathrm{d}^{1}$ configuration) characterized by a $\mathbf{g}$ tensor with small $g$ anisotropy with a well-resolved hyperfine interaction with the ${ }^{51} \mathrm{~V}$ nucleus $(I=7 / 2)$. While the X-band CW-EPR specrum can be simulated in terms of a single species (Figure 2a), the W-band CW-EPR spectrum (Figure 2c) shows an unidentified signal at low field and the $g_{\|}$features seem to be split in different components. This may be due to impurities, but could also reflect the difference between the presence or absence of an axial ligand (see later). The spin Hamiltonian values used for the spectral simulation of the dominant form are given in Table 1.

Upon dissolution of $\mathrm{F}_{64} \mathrm{PcVO}$ in ethanol, the EPR parameters change compared to the powder (Figure 3, Table 1). The heterogeneity observed for the EPR spectrum of the powder is no longer present. The ESE-detected EPR spectra at X-and W-band of the frozen ethanol solution of $\mathrm{F}_{64} \mathrm{PcVO}$ can be simulated assuming an axial $\mathbf{g}$ and ${ }^{51} \mathrm{~V}$ hyperfine tensor (Figure 3 and Table 1). The change in the EPR spectra may stem 


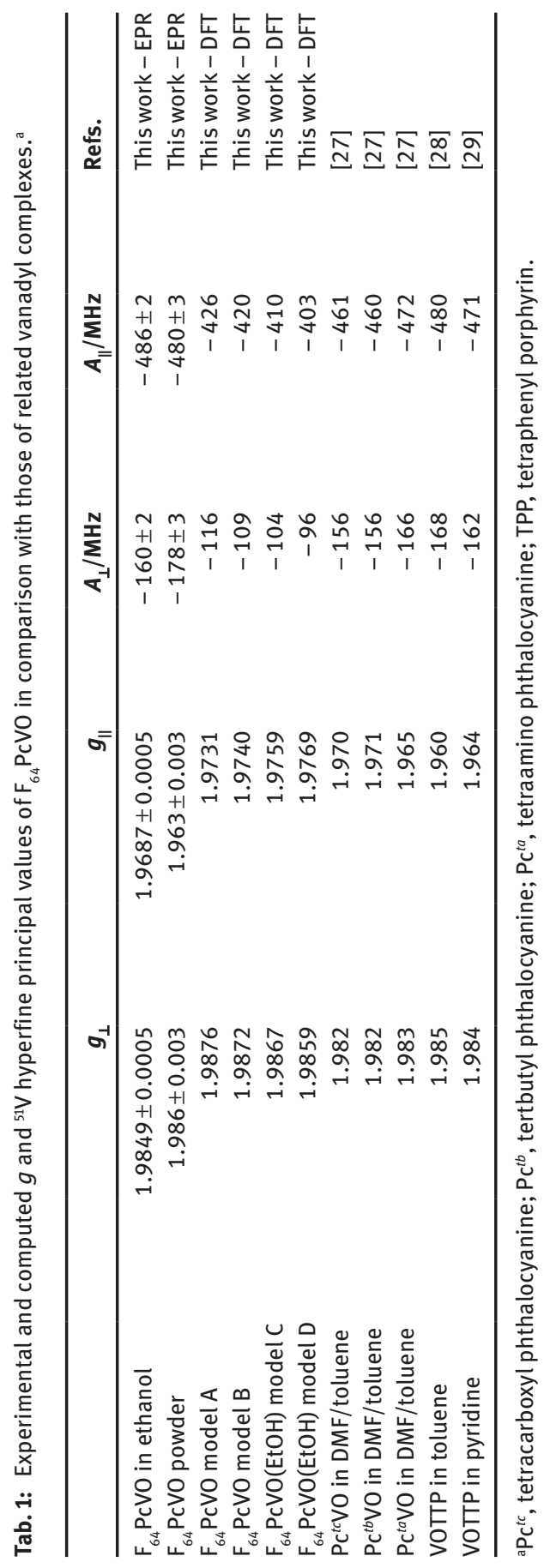



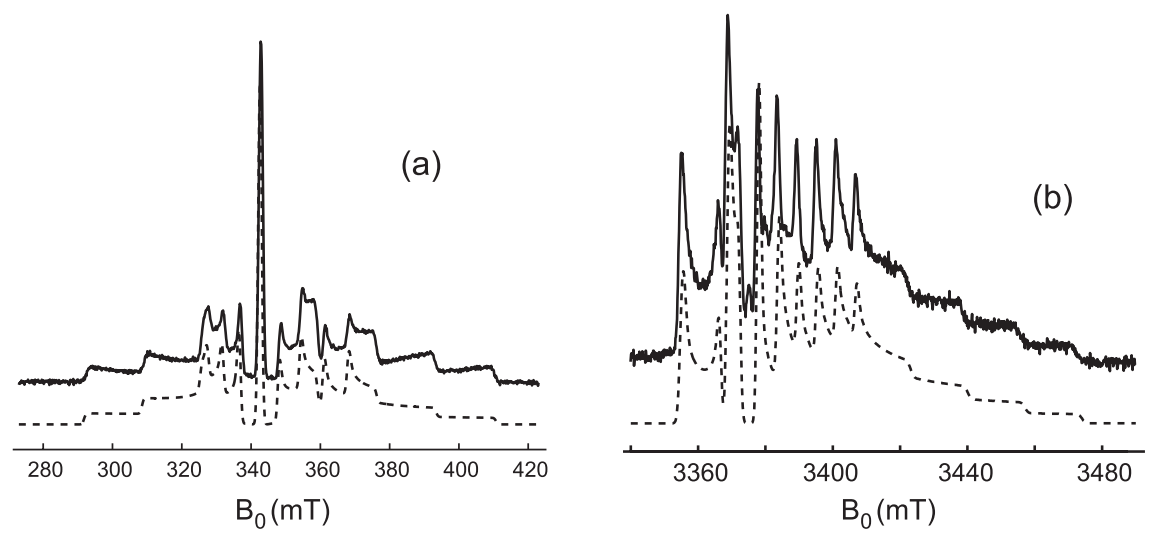

Fig. 3: Experimental (solid line) and simulated (dashed line) (a) X-band and (b) W-band ESEdetected EPR spectrum of a frozen ethanol solution of $\mathrm{F}_{64} \mathrm{PcVO}$ recorded at $10 \mathrm{~K}$. The simulation parameters are given in Table 1.

from a change in the dielectric constant of the matrix (pure $\mathrm{F}_{64} \mathrm{PcVO}$ compared to ethanol), but may also indicate axial ligation of ethanol to the metallo phthalocyanine. Indeed, earlier work established a relation between the coordination number and the $\mathbf{g}$ and ${ }^{51} \mathrm{~V}$ hyperfine tensor of $\left.[\mathrm{V}=\mathrm{O}]\right]^{2+}$ complexes [30, 31]

$$
\begin{aligned}
g_{\|} & =g_{e}-\frac{8 \lambda \alpha^{2}}{\Delta E_{\|}} \\
g_{\perp} & =g_{e}-\frac{2 \lambda \alpha^{2}}{\Delta E_{\perp}} \\
A_{\perp}-A_{\|} & =P\left(\frac{6}{7} \alpha^{2}-\left(g_{\|}-g_{e}\right)+\frac{6}{7}\left(g_{\perp}-g_{e}\right)\right)
\end{aligned}
$$

with $\lambda$ the spin-orbit coupling constant of the free $[\mathrm{V}=\mathrm{O}]^{2+}$ ion, $\alpha$ the coefficient of the $\mathrm{d}_{x y}$ orbital in the anti-bonding $\mathrm{b}_{2 g}^{*}$ orbital, $P=\mu_{B} u_{N} g_{2} g_{n}\left\langle r^{-3}\right\rangle$ the dipolar hyperfine coupling parameter of the unpaired electron, $\Delta E_{\|}=E\left(d_{x^{2}-y^{2}}\right)-E\left(d_{x y}\right)$ and $\Delta E_{\perp}=E\left(d_{x y y z}\right)-E\left(d_{x y}\right)$ in first-order approximation. A change in coordination number from 6 to 5 is expected to result in a decrease of $\Delta E_{\|}$and an increase of $\Delta E_{\perp}$. The former is caused by a decrease of repulsive interaction between the $\mathrm{d}_{x y}$ orbital and the corresponding orbitals of the four nitrogen atoms, the latter is caused by an increase of the $\mathrm{V}=\mathrm{O}$ bond strength. Thus, hexa-coordination should result in a larger $g_{\|}$value and smaller $g_{\perp}$ and $A_{\perp}-A_{\|}$values compared to the penta-coordinated conformation. This trend has been found experimentally for VOTPP upon ligation of pyridine (Table 1, [28, 29]). The principal $g$-values of 
the $\mathrm{F}_{64} \mathrm{PcVO}$ powder and the frozen solution of $\mathrm{F}_{64} \mathrm{PcVO}$ in ethanol also follow this trend (Table 1), but the value of $A_{\perp}-A_{\|}$shows an inverse trend (it increases after addition of ethanol). Nevertheless, the individual trends of $A_{\perp}$ and $A_{\|}$follow those observed for VOTPP and hence suggest that axial ligation of ethanol has occurred.

To corroborate the EPR studies, the dependency of the electronic absorption on concentration is studied to reveal possible aggregation (Figure 4, and Figures S1-S2 in supporting information). The aggregation studies were limited by the onset of saturation above absorbance of 1, even when using a 1-mm cell, the highest concentration being about $1 \mathrm{mM}$. The data is less precise at the initial concentration and the Lambert-Beer law appears to follow a non-linear pattern, but the absorption-concentration dependency becomes linear at higher concentrations, up to the onset of saturation. Thus, the UV-Vis data support the presence of a single species.

In order to corroborate the hypothesis of axial ligation, pulsed EPR experiments were performed. When comparing the Mims ENDOR spectra of $\mathrm{F}_{64} \mathrm{PcVO}$ dissolved in ethanol and in deuterated ethanol taken at an observer position known to excite all orientations (central peak in Figure 2a), the signals due to the hyperfine interaction with the ethanol protons can be easily distinguished from those of the ${ }^{19} \mathrm{~F}$ nuclei (Figure 5). The maximum ${ }^{1} \mathrm{H}$ hyperfine coupling is about 3.5 $\mathrm{MHz}$. This is also confirmed by HYSCORE experiments (not shown).

To verify whether the observed trends in $g$ and ${ }^{51} \mathrm{~V}$ hyperfine values and the magnitude of the observed proton coupling agrees with axial coordination of ethanol, DFT computations were done on different models. Model A is
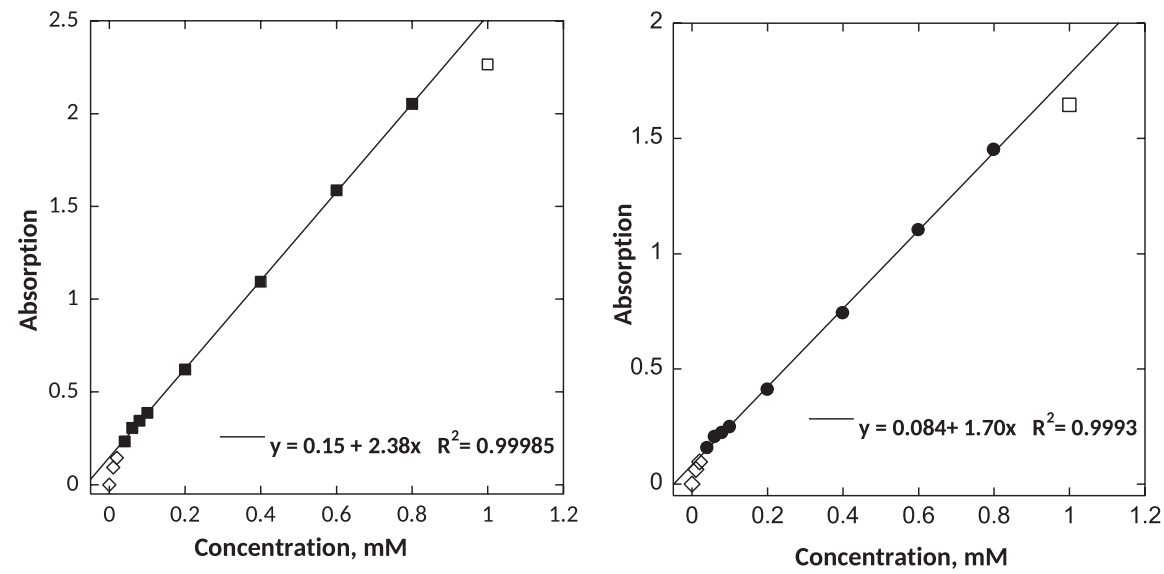

Fig. 4: Absorption versus concentration recorded (left) at $396 \mathrm{~nm}$ and (right) at $324 \mathrm{~nm}$, in a $1 \mathrm{~mm}$ cell. The three lowest $(\diamond)$ and highest $(\square)$ values have been excluded from the fits. 


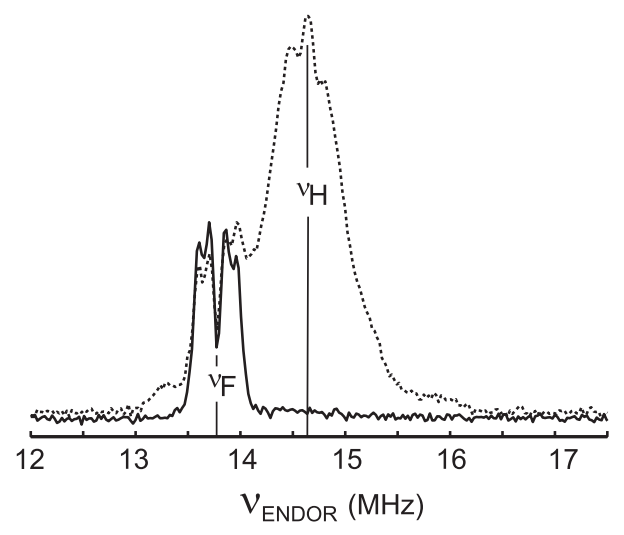

Fig. 5: Mims ENDOR spectra of a frozen solution of $\mathrm{F}_{64} \mathrm{PcVO}$ in ethanol (dotted line) and in deuterated ethanol (solid line), taken at the observer position corresponding to $g=g_{\text {iso }}\left(\mathrm{B}_{0}=343.6\right.$ $\mathrm{mT}) . v_{F}$ and $v_{H}$ are the Larmor frequencies of the ${ }^{19} \mathrm{~F}$ and ${ }^{1} \mathrm{H}$ nuclei, respectively.

the unligated $\mathrm{F}_{64} \mathrm{PcVO}$ complex in vacuum, model $\mathrm{B}$ is unligated $\mathrm{F}_{64} \mathrm{PcVO}$ considering the dielectric surrounding of ethanol. Models $\mathrm{C}$ and $\mathrm{D}$ are the $\mathrm{F}_{64} \mathrm{PcVO}$ complex ligated with one ethanol molecule, in vacuum and in the dielectric surrounding of ethanol, respectively. The vanadium coordination spheres of the DFT-optimized structures for $\mathrm{F}_{64} \mathrm{PcVO}$ are shown in comparison with the x-ray structure of $\mathrm{F}_{64} \mathrm{PcVO}\left(\mathrm{H}_{2} \mathrm{O}\right)$ [8] in Figure $1 \mathrm{~b}-\mathrm{d}$. The corresponding comparison of the metal coordination spheres parameters is listed in Table 2. The correlation between observed and calculated parameters are deemed satisfactory, especially since the computations reflect the expected geometric variations. The $\mathrm{V}-\mathrm{N}$ distances decrease upon the increase of the coordination number in line with a significant decrease, $33 \%$, in the deviation of the $\mathrm{V}$ from the least-squares plane, labeled $\mathrm{N}_{4}$, defined by the four coordinating $\mathrm{N}$. The larger distance between $\mathrm{V}-\mathrm{O}$ theoretically predicted for the ethanol-ligation case may be due to a larger steric hindrance than in the water-ligation case. The above information, taken together, lends credence to the suitability of the DFT models as the basis for interpreting the observed spectroscopic properties.

The computed $g$ and ${ }^{51} \mathrm{~V}$ hyperfine values are given in Table 1 . The agreement with the experiment is satisfactory, but not perfect. This is expected, since state-of-the-art DFT computations are still not able to reproduce exactly the $g$ and metal hyperfine values of transition-metal complexes [32]. Comparison of model $\mathrm{A}\left(\mathrm{F}_{64} \mathrm{PcVO}\right.$ in vacuum) and model $\mathrm{C}\left(\mathrm{F}_{64} \mathrm{PcVO}(\mathrm{EtOH})\right.$ in vacuum $)$ shows that the DFT-computed EPR values follow the predicted trends for the change from pentato hexacoordination [see Eqs. (1)]. However, the computations also indicate that the dielectric constant of the matrix matters and induces changes in the $g$ and 
Tab. 2: Structural parameters (in $\AA$ ) of the vanadium coordination sphere. $\mathrm{CN}=$ coordination number.

\begin{tabular}{|c|c|c|c|}
\hline & $\begin{array}{l}\text { DFT Model A } \\
\mathrm{N}_{4} \mathrm{~V}=0 \\
\text { CN } 5\end{array}$ & $\begin{array}{l}\text { DFT Model D } \\
\mathrm{N}_{4}(\mathrm{EtOH}) \mathrm{V}=0 \\
\mathrm{CN} 6\end{array}$ & $\begin{array}{r}\text { Experimental } \\
\mathrm{N}_{4}\left(\mathrm{H}_{2} \mathrm{O}\right) \mathrm{V}=0 \\
\mathrm{CN} 6\end{array}$ \\
\hline V-N & 2.058 & 2.045 & $2.027(4)$ \\
\hline $\mathrm{V}=0$ & 1.583 & 1.598 & $1.586(4)$ \\
\hline $\mathrm{V}-\mathrm{O}\left(\mathrm{EtOH}\right.$ or $\left.\mathrm{H}_{2} \mathrm{O}\right)$ & - & 2.439 & $2.358(4)$ \\
\hline V-N ${ }_{4}$ plane & 0.552 & 0.371 & $0.355(5)$ \\
\hline Ref. & This work & This work & [8] \\
\hline
\end{tabular}

${ }^{51} \mathrm{~V}$ hyperfine values (compare model $\mathrm{A}$ and $\mathrm{B}$, and $\mathrm{C}$ and $\mathrm{D}$, Table 1). The proton couplings calculated from the DFT computations for the acidic ethanol proton are overestimated (Table 3) with a maximum coupling of 5.9 (6.4) MHz for model C (D) compared to the maximum of $3.5 \mathrm{MHz}$ obtained experimentally. This suggests that the relative orientation of the ethanol versus $\mathrm{F}_{64} \mathrm{PcVO}$ is in the frozen solution slightly different than estimated by the computations. This is not unlikely given that the surrounding solvent molecules will interact with the ligating molecule. This was also observed for in X-ray structures of $\mathrm{F}_{64} \mathrm{PcVO}\left(\mathrm{H}_{2} \mathrm{O}\right)$ where the presence of acetone or methyl ethyl ketone was found to lead to different long range ordering, but only small differences in the $\mathrm{F}_{64} \mathrm{PcVO}-\mathrm{OH}_{2}$ distance. Moreover, for $\mathrm{F}_{64} \mathrm{PcCu}$ in ethanol, a maximum proton hyperfine coupling of $4.1 \mathrm{MHz}$ could be ascribed to the acidic proton of a coordinated ethanol, close to the value observed here for $\mathrm{F}_{64} \mathrm{PcVO}(\mathrm{EtOH})[7]$. The acidity of the $\mathrm{R}-\mathrm{OH}$ proton is exacerbated by the enhanced Lewis acidity of the vanadium, induced by the strong electron width-drawing effects of the fluoro substituents, but neither EPR nor UV-Vis data suggest the presence of a coordinated ethoxide anion. Assuming that the observed change in maximum proton coupling is only due to a changed dipolar interaction (Fermi-contact term is neglible) and taking into account the difference in principal $g$ values, the $\mathrm{V}-\mathrm{H}$ distance in $\mathrm{F}_{64} \mathrm{PcVO}(\mathrm{EtOH})$ is $\sim 0.15 \AA$ longer than the $\mathrm{Cu}-\mathrm{H}$ distance in $\mathrm{F}_{64} \mathrm{PcCu}(\mathrm{EtOH})$. Note also that the DFT computations

Tab. 3: DFT computed principal hyperfine values of the acidic proton of ethanol in $\mathrm{F}_{64} \mathrm{PcVO}(\mathrm{EtOH})$ models $\mathrm{C}$ and $\mathrm{D}$.

\begin{tabular}{lrrr}
\hline & $\boldsymbol{A}_{x} / \mathbf{M H z}$ & $\boldsymbol{A}_{y} / \mathbf{M H z}$ & $\boldsymbol{A}_{z} / \mathbf{M H z}$ \\
\hline Model C & -2.8 & -2.9 & 5.9 \\
Model D & -3.0 & -3.1 & 6.4 \\
\hline
\end{tabular}


predict a $\mathrm{V}=\mathrm{O}$ distance of $1.59 \AA$ for all models (Table 2), in line with the earlier experimental observation that the $\mathrm{V}=\mathrm{O}$ distance is insensitive to the metal coordination environments and hydrogen bonding [8].

Figure 5 also reveals a ${ }^{19} \mathrm{~F}$ hyperfine coupling of $\sim[-0.4-0.40 .8] \pm 0.1 \mathrm{MHz}$. The dipolar interaction expected theoretically for the aromatic fluorine atoms on the non-peripheral positions is [- $0.35-0.350 .71] \mathrm{MHz}(\mathrm{V}-\mathrm{F}$ distance of 6.06 $\AA$ ). Hence, the observed fluorine nuclear interactions are predominantly dipolar in nature and quasi no Fermi-contact contributions are found on the ${ }^{19} \mathrm{~F}$ nuclei. A similar observation was made earlier for $\mathrm{F}_{64} \mathrm{PcCu}$ [7].

Finally, also the X-band ${ }^{14} \mathrm{~N}$ HYSCORE spectra of the frozen ethanol solution of $\mathrm{F}_{64} \mathrm{PcVO}$ (Figure 6a, c, and e) were recorded. Clear cross peaks due to the
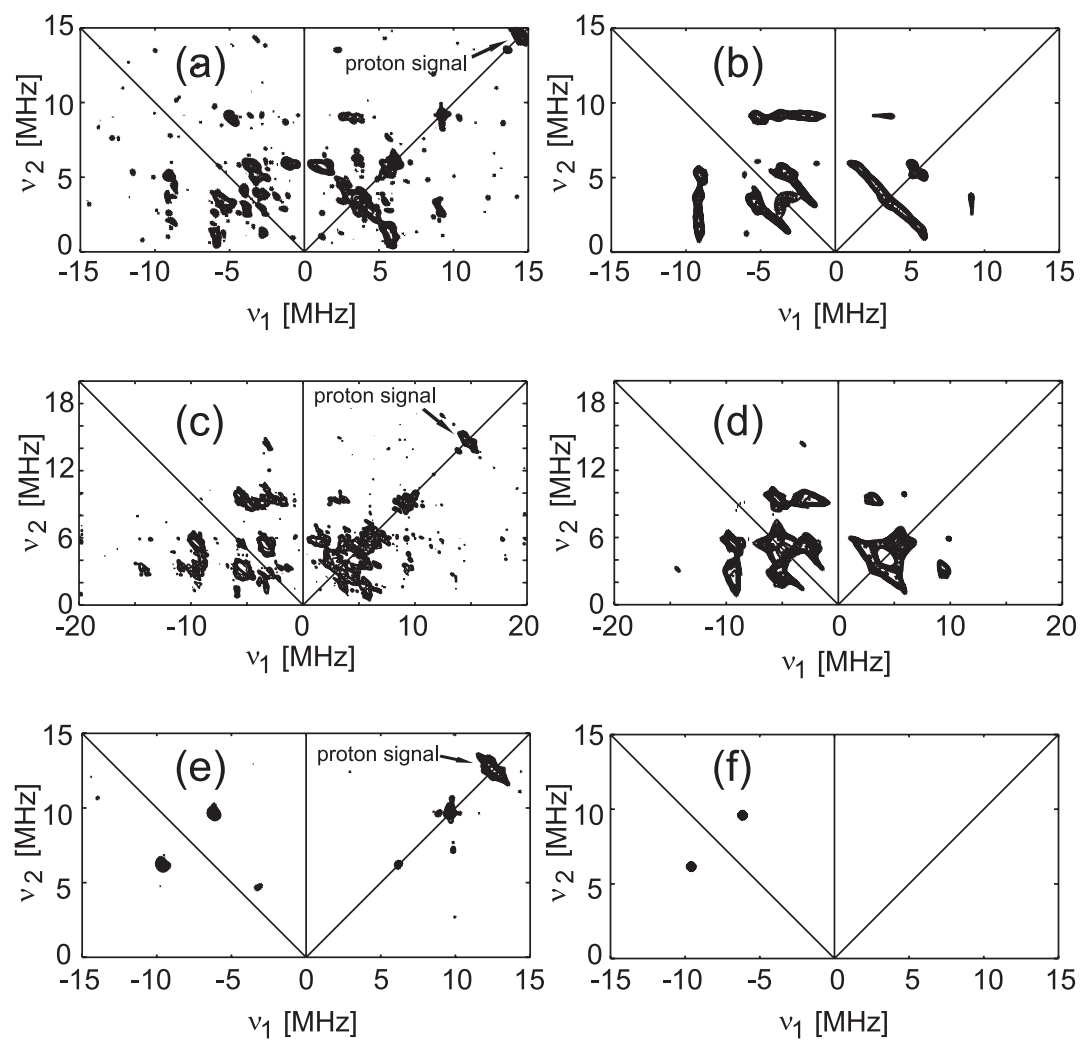

Fig. 6: Experimental (a, c, e) and simulated (b, d, f) X-band ${ }^{14} \mathrm{~N}$ HYSCORE spectra of a frozen ethanol solution of $\mathrm{F}_{64} \mathrm{PcVO}$ recorded at $10 \mathrm{~K}$ at an observer positions corresponding with (a, b) $g=g_{\perp}\left(\mathrm{B}_{0}=338.1 \mathrm{mT}\right),(\mathrm{c}, \mathrm{d}) g=g_{\text {iso }}\left(\mathrm{B}_{0}=342.8 \mathrm{mT}\right)$, (e, f) $g=g_{\|,}, m_{l}=7 / 2\left(\mathrm{~B}_{0}=293.9 \mathrm{mT}\right)$. The used $\tau$ values were $96 \mathrm{~ns}$ and $176 \mathrm{~ns}$. The simulation parameters are given in Table 3. 
hyperfine and nuclear quadrupole interactions of the coordinating isoindole nitrogen nuclei are observed. The spectra can be satisfactorily simulated using the spin Hamiltonian values given in Table 4 (Figure 6b, $d$, and f). The values of the ${ }^{14} \mathrm{~N}$ hyperfine interaction computed by DFT for the different models agree quite well with the experimental values (little influence of the matrix on the nitrogen hyperfine and quadrupole principal values could be found) and also resemble those experimentally found for similar [V(IV)O $]^{2+}$-containing phthalocyanine and porphyrin complexes (Table 4). Interestingly, Table 2 reveals a sensitivity of the $\mathrm{V}-\mathrm{N}$ bond distances to changes in the metal coordination number.

Some interesting observations can be made about the ${ }^{14} \mathrm{~N}$ quadrupole coupling tensors of the metal-bound nitrogens of the vanadyl phthalocyanine and porphyrin complexes (Table 4). First, in all cases, the component along the $z$ axis (normal to the macrocycle) is small in comparison to the other two principal values (in absolute value). This large anisotropy of the quadrupole tensor is related to the typical relatively flat structure of the macrocycle and the fact that the in-plane axes are differentiated due to the presence of the metal and independent of the nature of the metal. Indeed, $\mathrm{F}_{64} \mathrm{PcCu}$ has an out-of-plane quadrupole value that is zero within the experimental error [7]. On first inspection of Table 4, the sign and relative order of the principle quadrupole components of the indole nitrogens of $\mathrm{Pc}^{t c} \mathrm{VO}$ and $\mathrm{Pc}^{t b} \mathrm{VO}$ seem to be totally different than for the other complexes. However, this is only an apparent large deviation. As was shown by Czjzek many years ago, the conventional presentation of nuclear quadrupole data conceals the continuous connection between regions of positive and negative values of the quadrupole's main component [34]. He introduced a conceptual view that clearly expresses the topological structure of the $\left(\frac{e^{2} q Q}{2 h}, \eta\right)$ -plane. For this, the quadrupole components in Table 4 need to be re-ordered and renamed to $V_{i i}(i=x, y, z)$, such that

$$
\left|V_{x x}\right| \leq\left|V_{y y}\right| \leq\left|V_{z z}\right|
$$

in which case

$$
V_{z z}=\frac{e^{2} q Q}{2 h}, \eta=\frac{V_{x x}-V_{y y}}{V_{z z}}
$$

with $0 \leq \eta \leq 1$ the asymmetry constant, $e Q$ the nuclear quadrupole constant and $e q$ the electric field gradient. Plotting now the $-2 V_{x x}$ values in function of $2\left|2 V_{z z}+V_{x x}\right| / \sqrt{3}$ for constant values of $\eta$ or $V_{z z}$ leads to a representation that reveals the continuous connection between areas of positive and negative $V_{z z}$ via the common boundary defined by $\eta=1$ (Figure 7 ). In series of compounds with 


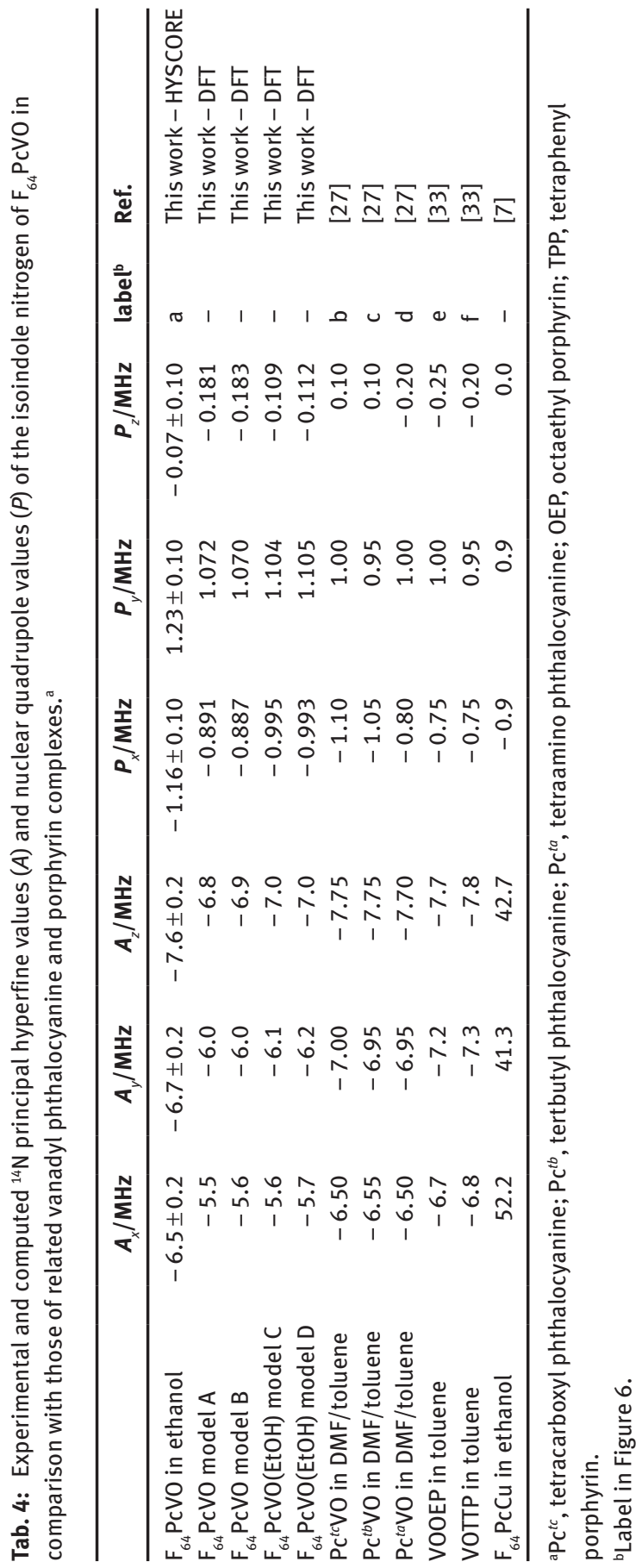




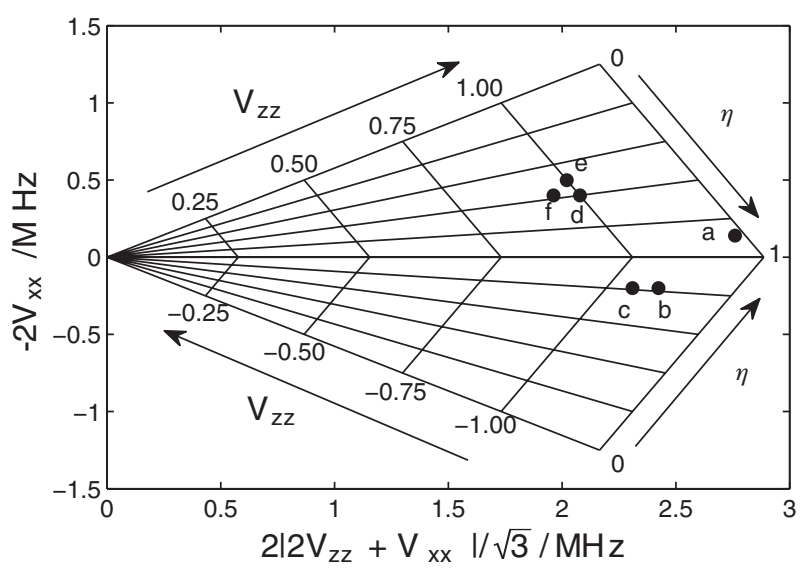

Fig. 7: Representation of the ${ }^{14} \mathrm{~N}$ nuclear quadrupole data of the vanadyl complexes indicated in Table 3 (labeling a-f) using a so-called Czjzek plot [34].

quadrupolar tensors characterized by $\eta$ values near 1 (such as the cases at hand), small changes in (the already small) $V_{x x}$ can induce a change in sign of $V_{x x}$, with the concomitant change in sign of the overall quadrupole tensor. While the effects on the quadrupole tensors seem at first sight drastic, the effects that induce them are not. The so-called Czjzek plot has the advantage that it clearly shows the continuation through the $\eta=1$ boundary. Czjzek plots are commonly used in other fields dealing with nuclear quadrupole tensors, such as time Differential Perturbed Angular Correlation (TDPAC) spectroscopy, but have, to our knowledge, never been employed in EPR spectroscopy despite their usefulness. Figure 7 represents such a Czjzek plot for the experimental quadrupole data from Table 4 and shows that the deviation of the quadrupole values of $\mathrm{Pc}^{t c} \mathrm{VO}$ and $\mathrm{Pc}^{\mathrm{tb}} \mathrm{VO}$ (points $\mathrm{b}$ and $\mathrm{c}$ ) from those of $\mathrm{F}_{64} \mathrm{PcVO}$ and $\mathrm{Pc}^{\text {ta }} \mathrm{VO}$ (point a and d, respectively) is not more drastic than the deviation between the data of these two latter phthalocyanines.

\section{Conclusions}

The reported multi-frequency CW and pulsed EPR experiments show clearly that the presence of the bulky and electron-withdrawing substituents in $\mathrm{F}_{64} \mathrm{PcVO}$ strongly influence the properties of the molecule. The $\mathrm{F}_{64} \mathrm{Pc}^{2-}$ ligand is not only one of the most electronic deficient ligands, but also a sterically bulky one. Thus, the EPR spectrum of the undiluted $\mathrm{F}_{64} \mathrm{PcVO}$ powder shows no major dipolar interactions, indicating single-site isolation. A similar behavior was previously found for 
the copper(II)-ligated variant of this molecule, $\mathrm{F}_{64} \mathrm{PcCu}$ [7]. W-band EPR revealed the presence of different species in the $\mathrm{F}_{64} \mathrm{PcVO}$ powder, of which one is strongly dominating. This heterogeneity may be due to the presence of $\mathrm{F}_{64} \mathrm{PcVO}$ with and without axial ligands, and/or may be due to a small fraction of spin-spin interacting vanadyl centers. The heterogeneity is fully lifted by dissolving the molecule in coordinating ethanol, which seems to exclude the presence of vanadyl impurities in the powder as a possible reason for the observed EPR heterogeneity, while UV-Vis data support the single-site isolation feature in solution. The observation of clear proton hyperfine couplings in the ENDOR spectra of $\mathrm{F}_{64} \mathrm{PcVO}$ in a frozen ethanol solution proves the occurrence of axial ethanol ligation. This axial ligation may aid the dissolution of the compound in the alcohol, a property that is of great importance for potential applications, especially in view of the current search for 'green' chemistry. Furthermore, the vanadyl center in $\mathrm{F}_{64} \mathrm{PcVO}$ is much more electron poor than that of related vanadyl centers due to the presence of the fluorinated substituents. This may explain its large affinity for axial ligation by Lewis bases as observed also for the $\mathrm{Cu}$ (II) and $\mathrm{Zn}$ (II) variants of the phthalocyanine $[7,35]$. The accessibility of the metal center to axial ligands is a prerequisite for use of this complex in homo- and or heterogeneous catalytic applications. Finally, the study reveals that the hyperfine and nuclear quadrupole data of the vanadium-bound nitrogens (isoindole or pyrrole) do not vary much when different vanadyl phthalocyanine and porphyrin complexes are compared. Apparent large changes in the nuclear quadrupole constant are in essence only due to small variations in the electronic structure as is better evaluated using a Czjzek plot.

Acknowledgement: Partial support from the National Science Foundation (SMG) is gratefully acknowledged. SVD thanks the Fund for Scientific Research - Flanders for support (Grant G.0687.13).

\section{References}

1. N. B. McKeown, Phthalocyanine Materials: Synthesis, Structure and Function, Cambridge University Press, Cambridge (1998).

2. A. B. P. L. C. C Leznoff (Ed.): Phthalocyanine: Properties and Applications, VCH Publishers, New York (1996).

3. H.-J. Lee, W. W. Brennessel, J. A. Lessing, W. W. Brucker, V. G. Young Jr, S. M. Gorun, Chem. Commun. (2003) 1576.

4. B. A. Bench, A. Beveridge, W. M. Sharman, G. J. Diebold, J. E. van Lier, S. M. Gorun, Angew. Chem. Int. Ed. 41 (2002) 748.

5. B. A. Bench, W. W. Brennessel, H.-J. Lee, S. M. Gorun, Angew. Chem. Int. Ed. 114 (2002) 750. 
6. C. Keil, O. Tsaryova, L. Lapok, C. Himcinschi, D. Wöhrle, O. Hild, D. Zahn, S. Gorun, D. Schlettwein, Thin Solid Films 517 (2009) 4379.

7. H. Moons, Ł. Łapok, A. Loas, S. Van Doorslaer, S. M. Gorun, Inorg. Chem. 49 (2010) 8779.

8. Ł. tapok, M. Lener, O. Tsaryova, S. Nagel, C. Keil, R. Gerdes, D. Schlettwein, S. M. Gorun, Inorg. Chem. 50 (2011) 4086.

9. W. Mims, Proc. R. Soc. London Ser. A 283 (1965) 452.

10. P. Höfer, A. Grupp, H. Nebenführ, M. Mehring, Chem. Phys. Lett. 132 (1986) 279.

11. S. Stoll, A. Schweiger, J. Magn. Reson. 178 (2006) 42.

12. F. Neese, J. Chem. Phys. 115 (2001) 11080.

13. F. Neese, J. Phys. Chem. A 105 (2001) 4290.

14. F. Neese, J. Chem. Phys. 118 (2003) 3939.

15. F. Neese, J. Chem. Phys. 122 (2005) 034107.

16. S. Sinnecker, A. Rajendran, A. Klamt, M. Diedenhofen, F. Neese, J. Phys. Chem. A 110 (2006) 2235.

17. J. P. Perdew, Phys. Rev. B 33 (1986) 8822.

18. J. P. Perdew, Phys. Rev. B 34 (1986) 7406.

19. A. D. Becke, Phys. Rev. A 38 (1988) 3098.

20. B. I. Dunlap, J. Connolly, J. Sabin, J. Chem. Phys. 71 (1979) 3396.

21. O. Vahtras, J. Almlöf, M. Feyereisen, Chem. Phys. Lett. 213 (1993) 514.

22. K. Eichkorn, O. Treutler, H. Öhm, M. Häser, R. Ahlrichs, Chem. Phys. Lett. 240 (1995) 283.

23. A. Schäfer, H. Horn, R. Ahlrichs, J. Chem. Phys. 97 (1992) 2571.

24. P. Stephens, F. Devlin, C. Chabalowski, M. J. Frisch, J. Phys. Chem. 98 (1994) 11623.

25. V. Barone, in: Recent Advances in Density Functional Methods - Part I, Recent Advantages in Computational Chemistry, Vol. 1, D. Chong (Ed.): World Scientific (1996), P. 287.

26. A. J. H. Wachters, J. Chem. Phys. 52 (1970) 1033.

27. K. Fukui, H. Ohya-Nishiguchi, H. Kamada, M. Iwaizumi, Y. Xu, Bull. Chem. Soc. Jpn. 71 (1998) 2787.

28. M. Sato, T. Kwan, Bull. Chem. Soc. Jpn. 47 (1974) 1353.

29. S. Sharma, A. Kumar, P. Chand, B. Sharma, S. Sarkar, Spectrochim. Acta Mol. Biomol. Spectrosc. 63 (2006) 556.

30. Y. Xu, S. Shi, Appl. Magn. Reson. 11 (1996) 1.

31. N. D. Chasteen, in: Biological magnetic resonance, edited by B. L. and R. J. Springer, (1981), P. 53.

32. F. Neese, Coord. Chem. Rev. 253 (2009) 526.

33. K. Fukui, H. Ohya-Nishiguchi, H. Kamada, J. Phys. Chem. 97 (1993) 11858.

34. G. Czjzek, Phys. Rev. B 25 (1982) 4908.

35. H. Moons, A. Loas, S. M. Gorun, S. Van Doorslaer, Dalton Trans. 43 (2014) 14942.

Supplemental Material: The online version of this article (DOI: 10.1515/zpch-2016-0827) offers supplementary material, available to authorized users. 